\title{
Fisher Fixed Point Results in Generalized Metric Spaces with a Graph
}

\author{
Karim Chaira, ${ }^{1}$ Abderrahim Eladraoui $\mathbb{D D}^{2}{ }^{2}$ Mustapha Kabil, ${ }^{1}$ and Abdessamad Kamouss $\mathbb{I D}^{1}$ \\ ${ }^{1}$ Laboratory of Mathematics and Applications, Faculty of Sciences and Technologies, University Hassan II of Casablanca, \\ P.O. Box 146, Mohammedia, Morocco \\ ${ }^{2}$ Laboratory of Algebra, Analysis and Applications, Faculty of Sciences Ben Msik, University of Hassan II Casablanca, Morocco
}

Correspondence should be addressed to Abdessamad Kamouss; akamouss@gmail.com

Received 3 September 2019; Revised 29 December 2019; Accepted 6 February 2020; Published 16 March 2020

Academic Editor: Nawab Hussain

Copyright (c) 2020 Karim Chaira et al. This is an open access article distributed under the Creative Commons Attribution License, which permits unrestricted use, distribution, and reproduction in any medium, provided the original work is properly cited.

We discuss Fisher's fixed point theorem for mappings defined on a generalized metric space endowed with a graph. This work should be seen as a generalization of the classical Fisher fixed point theorem. It extends some recent works on the enlargement of Banach Contraction Principle to generalized metric spaces with graph. An example is given to illustrate our result.

\section{Introduction and Preliminaries}

Currently, fixed point theory is a very active area of research because of its applications in multiple fields. It concerns the results which indicate that, under certain conditions, selfmapping on a set admits a fixed point. Among all the results in metric fixed point theory, the Banach Contraction Principle [1] is the most celebrated one due to its simplicity and ease of application in major areas of mathematics. Subsequently, many authors extend and generalize this principle in different directions. In 1981, Fisher [2] proved the following related fixed-point theorem involving two mappings on two complete metric spaces as follows.

Theorem 1. Let $(X, d)$ and $(Y, \delta)$ be complete metric spaces. If $T$ is a mapping from $X$ into $Y$ and $S$ is a mapping from $Y$ into $X$, satisfying the following conditions,

$$
\left\{\begin{array}{l}
\delta(T x, T S y) \leq c \max \{d(x, S y), \delta(y, T x), \delta(y, T S y)\} \\
d(S y, S T x) \leq c \max \{\delta(y, T x), d(x, S y), d(x, S T x)\}
\end{array}\right.
$$

for all $x$ in $X$ and $y$ in $Y$ where $0 \leq c<1$, then ST has a unique fixed point $z$ in $X$ and TS has a unique fixed point $w$ in $Y$. Furthermore, $T z=w$ and $S w=z$.
In 2017, Chaira et al. [3] extend the Fisher theorem in the setting of partially ordered generalized metric spaces.

The concept of standard metric space is a fundamental tool in topology, functional analysis, and nonlinear analysis. In recent years, several generalizations of standard metric space have appeared. In 1993, Czerwik [4] introduced the concept of a b-metric space. Since then, several works have dealt with fixed point theory in such spaces. In 2000, Hitzler and Seda [5] introduced the notion of dislocated metric spaces in which self-distance of a point need not be equal to zero. Such spaces play a very important role in topology and logical programming (see [6]). In this work, we present a new generalized metric space introduced by Jleli and Samet in [7] and that recovers a large class of topological spaces including standard metric spaces, b-metric spaces, dislocated metric spaces, and modular spaces with the Fatou property. Also, several interesting results about the existence and the uniqueness of the fixed point were proved in the setting of this generalized metric space (see [3, 7-13]).

An interesting approach in the theory of the fixed point in some general structures was recently given by Jachymski [14] in the setting of metric spaces endowed with a graph and Samet and Turinici [15] in the setting of metric spaces endowed with an arbitrary binary relation. 
In this work, inspired by the ideas given in [14-18], we investigate Fisher fixed-point theorem in generalized metric spaces with a graph. As corollary, we obtain Fisher fixed-point theorem in the setting of standard metric spaces endowed with a graph. An example is provided to illustrate our result.

In the following, we describe the mathematical background materials which are necessary for establishing the results in this paper. A directed graph or digraph $G$ is determined by a nonempty set $V(G)$ of its vertices and the set $E(G) \subset V(G) \times$ $V(G)$ of its arcs. Let $\Delta$ denote the diagonal of the Cartesian product $V(G) \times V(G)$. A digraph is said to be reflexive if the set $E(G)$ of its arcs contains all loops, i.e., $\Delta \subset E(G)$. $G$ is said to be transitive whenever for any $x, y, z \in V(G)$ :

$$
[(x, y) \in E(G) \text { and }(y, z) \in E(G)] \text { implies }(x, z) \in E(G) \text {. }
$$

We say that a vertex $x$ in $V(G)$ is isolated if for any vertex $y$ in $V(G)$ such that $x \neq y$, and we have neither $(x, y) \in E(G)$ nor $(y, x) \in E(G)$.

By $G^{-1}$ we denote the converse of a digraph $G$, that is, the digraph obtained from $G$ by reversing the direction of arcs. Thus, we have

$$
E\left(G^{-1}\right)=\{(x, y) \in V(G) \times V(G):(y, x) \in E(G)\} .
$$

Also, $\widetilde{G}$ denotes the undirected graph obtained from $G$ by ignoring direction of the edges. Thus, we have

$$
E(\widetilde{G})=E(G) \cup E\left(G^{-1}\right) .
$$

Given a digraph $G=(V, E)$, a (di)path of $G$ is a sequence $\left(a_{0}, a_{1}, \ldots, a_{n}\right)$ with $\left(a_{i}, a_{i+1}\right) \in E(G)$, for each $i \in \mathbb{N}$. A finite path $\left(a_{0}, a_{1}, \ldots, a_{n}\right)$ is said to have length $n$, for $n \in \mathbb{N}$. A closed directed path of length $n>1$ from $x$ to $y$, i.e., $x=y$, is called a directed cycle. An acyclic digraph is a digraph that has no directed cycle. A digraph is connected if there is a finite (di)path joining any two of its vertices and it is weakly connected if $\widetilde{G}$ is connected.

If $G$ is such that $E(G)$ is symmetric and $x$ is a vertex in $G$, then the subgraph $G_{x}$ consisting of all edges and vertices, which are contained in some path beginning at $x$, is called the component of $G$ containing $x$. In this case, $V\left(G_{x}\right)=[x]_{G}$, where $[x]_{G}$ is the equivalence class of the following relation $R$ defined on $V(G)$ by the rule $y R z$ if there is a path in $G$ from $y$ to $z$. Clearly, $G_{x}$ is connected. We say that two vertices $x$ and $y$ in $V(G)$ are connected if $y \in[x]_{G}$ or $x \in[y]_{G}$.

The basic concepts related to a graph may be found in any textbook on graph theory, see for example $[19,20]$.

Definition 1. A sequence $\left\{x_{n}\right\}$ in $V(G)$ is said to be

(i) $G$-increasing, if $x_{n+1} \in\left[x_{n}\right]_{G}$, for all $n \in \mathbb{N}$;

(ii) G-decreasing, if $x_{n} \in\left[x_{n+1}\right]_{G}$, for all $n \in \mathbb{N}$;

(iii) $G$-monotone, if it is either $G$-increasing or G-decreasing

Let $X$ be a nonempty set and $\mathscr{D}: X \times X \longrightarrow[0,+\infty]$ be a given mapping. For every $x \in X$, let us define the set
$\mathscr{C}(\mathscr{D}, X, x)=\left\{\left\{x_{n}\right\} \subset X: \lim _{n \longrightarrow \infty} \mathscr{D}\left(x_{n}, x\right)=0\right\} . \quad$ The mapping $\mathscr{D}$ is called a generalized metric on $X$ if it satisfies the following conditions [7]:

$\left(\mathscr{D}_{1}\right)$ for every $(x, y) \in X \times X, \mathscr{D}(x, y)=0 \Longrightarrow x=y$. $\left(\mathscr{D}_{2}\right)$ for every $(x, y) \in X \times X, \mathscr{D}(x, y)=\mathscr{D}(y, x)$.

$\left(\mathscr{D}_{3}\right)$ there exists $C>0$ such that if $(x, y) \in X \times X$ and $\left\{x_{n}\right\} \in \mathscr{C}(\mathscr{D}, X, x)$, then

$$
\mathscr{D}(x, y) \leq C \limsup _{n \longrightarrow \infty} \mathscr{D}\left(x_{n}, y\right) .
$$

In this case, we say that the pair $(X, \mathscr{D})$ is a generalized metric space. Obviously, if the set $\mathscr{C}(\mathscr{D}, X, x)$ is empty for every $x \in X$, then $(X, \mathscr{D})$ is a generalized metric space if and only if $\left(\mathscr{D}_{1}\right)$ and $\left(\mathscr{D}_{2}\right)$ are satisfied. A sequence $\left\{x_{n}\right\}$ in a generalized metric space $(X, \mathscr{D})$ is said to be $\mathscr{D}$-convergent to $x \in X$ if $\left\{x_{n}\right\} \in \mathscr{C}(\mathscr{D}, X, x)$. Note that if the set $\mathscr{C}(\mathscr{D}, X, x)$ is not empty for some $x \in X$, then $\mathscr{D}(x, x)=0$. A sequence $\left\{x_{n}\right\}$ is said to be a $\mathscr{D}$-Cauchy sequence if $\lim _{m, n \longrightarrow \infty} \mathscr{D}\left(x_{n}, x_{m}\right)=0$. Note that in a generalized metric space, a sequence has at most one limit and a $\mathscr{D}$-convergent sequence may not be $\mathscr{D}$-Cauchy sequence (see [16]). Moreover, $(X, \mathscr{D})$ is said to be $\mathscr{D}$-complete if every $\mathscr{D}$-Cauchy sequence in $X$ is $\mathscr{D}$-convergent to some element in $X$.

Definition 2. We say that $(X, \mathscr{D}, G)$ is $G$-regular if any $G$ increasing sequence (resp. G-decreasing sequence) $\left\{x_{n}\right\}$ which $\mathscr{D}$-converges to some $x \in V(G)$, we have $x \in\left[x_{n}\right]_{G}$ (resp. $x_{n} \in[x]_{G}$ ), for any $n \in \mathbb{N}$.

\section{Main Results}

Let $(X, \mathscr{D})$ and $(Y, \Delta)$ be two generalized metric spaces such that $X$ is endowed with a digraph $G$. Consider a nondecreasing function $\alpha:[0,+\infty) \longrightarrow[0,1)$ such that $\lim \sup _{t \rightarrow 0^{+}} \alpha(t)<\inf \{1,(1 / C)\}$ with $C$ as the positive real appeared in the condition $\left(\mathscr{D}_{3}\right)$ in the definition of the generalized metric $\mathscr{D}$.

Let $T: X \longrightarrow Y$ and $S: Y \longrightarrow X$ be two mappings and denote, for any $x_{0} \in X$,

$$
\begin{aligned}
\delta\left(S, T, x_{0}, \mathscr{D}, \Delta\right)= & \sup \left\{\mathscr{D}\left((S T)^{i} x_{0},(S T)^{j} x_{0}\right),\right. \\
& \left.\Delta\left(T(S T)^{i} x_{0}, T(S T)^{j} x_{0}\right): i, j \in \mathbb{N}\right\} .
\end{aligned}
$$

Theorem 2. Let $T: X \longrightarrow Y$ and $S: Y \longrightarrow X$ be two mappings satisfying the following conditions:

(1) Let

$$
\left\{\begin{array}{l}
\mathscr{D}(S y, S T x) \leq \alpha(\Delta(y, T x)) \max \{\mathscr{D}(x, S y), \Delta(y, T x), \mathscr{D}(x, S T x)\}, \\
\Delta(T x, T S y) \leq \alpha(\mathscr{D}(x, S y)) \max \{\mathscr{D}(x, S y), \Delta(y, T x), \Delta(y, T S y)\},
\end{array}\right.
$$

for any $x$ in $X$ and $y$ in $Y$ such that $x$ and Sy are connected. 
(2) $(X, \mathscr{D}, G)$ is $\mathscr{D}$-complete and G-regular.

(3) There exists an element $x_{0} \in X$ such that $\delta\left(S, T, x_{0}, \mathscr{D}, \Delta\right)<\infty$ and $(S T)^{i+1} x_{0} \in\left[(S T)^{i} x_{0}\right]_{G}$, for all $i \in \mathbb{N}$.

Then, the sequence $\left\{(S T)^{n} x_{0}\right\} \mathscr{D}$-converges to some $x^{*}$ in $X$. If $\mathscr{D}\left(x^{*}, S T x^{*}\right)<\infty$, then there exists $y^{*} \in Y$ such that $y^{*}=T x^{*}$ and $S y^{*}=x^{*}$; then, ST $x^{*}=x^{*}$ and $T S y^{*}=y^{*}$. Moreover, $\mathscr{D}\left(x^{*}, x^{*}\right)=0$ and $\Delta\left(y^{*}, y^{*}\right)=0$.

Proof. Step 1. Suppose that there exists an element $x_{0} \in X$ such that $\delta\left(S, T, x_{0}, \mathscr{D}, \Delta\right)<\infty$ and

$$
(S T)^{i+1} x_{0} \in\left[(S T)^{i} x_{0}\right]_{G}, \quad \text { for all } i \in \mathbb{N} \text {. }
$$

Consider the two sequences $\left\{x_{n}\right\} \subset X$ and $\left\{y_{n}\right\} \subset Y$ defined by

$$
\begin{gathered}
x_{n+1}=S T x_{n}, \\
y_{n}=T x_{n},
\end{gathered}
$$

for all $n \in \mathbb{N}$.

In (7), by taking $x=x_{p}$ and $y=y_{q}$, where $p$ and $q$ in $\mathbb{N}$, we obtain

$$
\left\{\begin{array}{l}
\mathscr{D}\left(S y_{q}, S T x_{p}\right) \leq \alpha\left(\Delta\left(y_{q}, T x_{p}\right)\right) \max \left\{\mathscr{D}\left(x_{p}, S y_{q}\right), \Delta\left(y_{q}, T x_{p}\right), \mathscr{D}\left(x_{p}, S T x_{p}\right)\right\} \\
\Delta\left(T x_{p}, T S y_{q}\right) \leq \alpha\left(\mathscr{D}\left(x_{p}, S y_{q}\right)\right) \max \left\{\mathscr{D}\left(x_{p}, S y_{q}\right), \Delta\left(y_{q}, T x_{p}\right), \Delta\left(y_{q}, T S y_{q}\right)\right\}
\end{array}\right.
$$

since $x_{p}$ and $S y_{q}=x_{q+1}$ are connected.

Thus,

$$
\left\{\begin{array}{l}
\mathscr{D}\left(x_{q+1}, x_{p+1}\right) \leq \alpha\left(\Delta\left(y_{q}, y_{p}\right)\right) \max \left\{\mathscr{D}\left(x_{p}, x_{q+1}\right), \Delta\left(y_{q}, y_{p}\right), \mathscr{D}\left(x_{p}, x_{p+1}\right)\right\} \\
\Delta\left(y_{p}, y_{q+1}\right) \leq \alpha\left(\mathscr{D}\left(x_{p}, x_{q+1}\right)\right) \max \left\{\mathscr{D}\left(x_{p}, x_{q+1}\right), \Delta\left(y_{q}, y_{p}\right), \Delta\left(y_{q}, y_{q+1}\right)\right\} .
\end{array}\right.
$$

In (11), by taking $p=q=n$, we obtain

$$
\left\{\begin{array}{l}
\mathscr{D}\left(x_{n+1}, x_{n+1}\right) \leq \alpha\left(\Delta\left(y_{n}, y_{n}\right)\right) \max \left\{\mathscr{D}\left(x_{n}, x_{n+1}\right), \Delta\left(y_{n}, y_{n}\right)\right\} \\
\Delta\left(y_{n}, y_{n+1}\right) \leq \alpha\left(\mathscr{D}\left(x_{n}, x_{n+1}\right)\right) \max \left\{\mathscr{D}\left(x_{n}, x_{n+1}\right), \Delta\left(y_{n}, y_{n}\right)\right\}
\end{array}\right.
$$

and by taking $p-1=q=n$, we obtain

$$
\left\{\begin{array}{l}
\mathscr{D}\left(x_{n+1}, x_{n+2}\right) \leq \alpha\left(\Delta\left(y_{n}, y_{n+1}\right)\right) \max \left\{\mathscr{D}\left(x_{n+1}, x_{n+1}\right), \Delta\left(y_{n}, y_{n+1}\right)\right\}, \\
\Delta\left(y_{n+1}, y_{n+1}\right) \leq \alpha\left(\mathscr{D}\left(x_{n+1}, x_{n+1}\right)\right) \max \left\{\mathscr{D}\left(x_{n+1}, x_{n+1}\right), \Delta\left(y_{n}, y_{n+1}\right)\right\}
\end{array}\right.
$$

For any $n$ in $\mathbb{N}$, let

It follows from inequalities (12) and (13) that

$$
\begin{aligned}
& d_{n}=\mathscr{D}\left(x_{n}, x_{n}\right), \\
& d_{n}^{\prime}=\mathscr{D}\left(x_{n}, x_{n+1}\right), \\
& s_{n}=\Delta\left(y_{n}, y_{n}\right), \\
& s_{n}^{\prime}=\Delta\left(y_{n}, y_{n+1}\right) .
\end{aligned}
$$

$$
\left\{\begin{array}{l}
d_{n+1} \leq \alpha\left(s_{n}\right) \max \left\{d_{n}^{\prime}, s_{n}\right\}, \\
s_{n}^{\prime} \leq \alpha\left(d_{n}^{\prime}\right) \max \left\{d_{n}^{\prime}, s_{n}\right\}, \\
d_{n+1}^{\prime} \leq \alpha\left(s_{n}^{\prime}\right) \max \left\{d_{n+1}, s_{n}^{\prime}\right\}, \\
s_{n+1} \leq \alpha\left(d_{n+1}\right) \max \left\{d_{n+1}, s_{n}^{\prime}\right\} .
\end{array}\right.
$$


Now for any $n$ in $\mathbb{N}$, let $k_{n}=\max \left\{\alpha\left(d_{n}^{\prime}\right), \alpha\left(s_{n}\right)\right\}$ and $t_{n}=\max \left\{\alpha\left(d_{n+1}\right), \alpha\left(s_{n}^{\prime}\right)\right\}$. System (15) becomes

$$
\left\{\begin{array}{l}
d_{n+1} \leq k_{n} a_{n}, \\
s_{n}^{\prime} \leq k_{n} a_{n}, \\
d_{n+1}^{\prime} \leq t_{n} b_{n}, \\
s_{n+1} \leq t_{n} b_{n},
\end{array}\right.
$$

where $a_{n}=\max \left\{d_{n}^{\prime}, s_{n}\right\} \quad$ and $\quad b_{n}=\max \left\{d_{n+1}, s_{n}^{\prime}\right\}$. Consequently,

$$
\left\{\begin{array}{l}
\max \left\{d_{n+1}, s_{n}^{\prime}\right\} \leq k_{n} a_{n}, \\
\max \left\{d_{n+1}^{\prime}, s_{n+1}\right\} \leq t_{n} b_{n} .
\end{array}\right.
$$

That is, $b_{n} \leq k_{n} a_{n}$ and $a_{n+1} \leq t_{n} b_{n}$, and so $a_{n+1} \leq k_{n} a_{n}$. Let $\left\{\delta_{n}\right\}$ be the sequence defined by $\delta_{n}=\delta\left(S, T,(S T)^{n} x_{0}, \mathscr{D}, \Delta\right)$, for any $n \in \mathbb{N}$. Since the function $\alpha$ is nondecreasing, $d_{n}^{\prime} \leq \delta_{0}$ and $s_{n} \leq \delta_{0}$, for any $n \in \mathbb{N}$,

$$
k_{n} \leq \alpha\left(\delta_{0}\right) \text { and so } a_{n+1} \leq \alpha\left(\delta_{0}\right) a_{n}, \quad \text { for any } n \in \mathbb{N},
$$

which implies that the sequences $\left\{a_{n}\right\}$ and $\left\{b_{n}\right\}$ converge to 0 . This yields

$$
\begin{aligned}
\lim _{n \longrightarrow \infty} \mathscr{D}\left(x_{n}, x_{n}\right) & =\lim _{n \longrightarrow \infty} \mathscr{D}\left(x_{n}, x_{n+1}\right) \\
& =\lim _{n \longrightarrow \infty} \Delta\left(y_{n}, y_{n}\right)=\lim _{n \longrightarrow \infty} \Delta\left(y_{n}, y_{n+1}\right)=0 .
\end{aligned}
$$

Step 2. Let us show that $\left\{x_{n}\right\}$ is a $\mathscr{D}$-Cauchy sequence. Let us fix $i$ and $j$ in $\mathbb{N}$.

For any $n \in \mathbb{N}, x=x_{n-1+j}$ and $S y=x_{n+i}$ are connected and by using the first inequality in (7), we have

$$
\begin{aligned}
\mathscr{D}\left((S T)^{n+i} x_{0},(S T)^{n+j} x_{0}\right) & =\mathscr{D}\left(x_{n+i}, x_{n+j}\right) \\
& \leq \alpha\left(\Delta\left(y_{n+i-1}, y_{n+j-1}\right)\right) \max \left\{\mathscr{D}\left(x_{n+j-1}, x_{n+i}\right), \Delta\left(y_{n+i-1}, y_{n+j-1}\right) \mathscr{D}\left(x_{n+j-1}, x_{n+j}\right)\right\} \\
& \leq \alpha\left(\Delta\left(y_{n+i-1}, y_{n+j-1}\right)\right) \delta_{n-1} .
\end{aligned}
$$

For any $n$ in $\mathbb{N}, x=x_{n+i}$ and $S y=x_{n+j}$ are connected and by using the second inequality in (7), we have

$$
\begin{aligned}
\Delta\left(T(S T)^{n+i} x_{0}, T(S T)^{n+j} x_{0}\right) & =\Delta\left(y_{n+i}, y_{n+j}\right) \\
& \leq \alpha\left(\mathscr{D}\left(x_{n+i}, x_{n+j}\right)\right) \max \left\{\mathscr{D}\left(x_{n+i}, x_{n+j}\right), \Delta\left(y_{n+i}, y_{n-1+j}\right) \Delta\left(y_{n-1+j}, y_{n+j}\right)\right\}, \\
& \leq \alpha\left(\mathscr{D}\left(x_{n+i}, x_{n+j}\right)\right) \delta_{n-1} .
\end{aligned}
$$

Thus, $\quad \delta_{n} \leq \alpha_{n} \delta_{n-1}, \quad$ where $\quad \alpha_{n}=\sup \left\{\alpha\left(\mathscr{D}\left(x_{n+i}\right.\right.\right.$, $\left.\left.\left.x_{n+j}\right)\right), \alpha\left(\Delta\left(y_{n+i-1}, y_{n+j-1}\right)\right): i, j \in \mathbb{N}\right\}$.

Since the function $\alpha$ is nondecreasing and $\mathscr{D}\left(x_{n+i}, x_{n+j}\right) \leq \delta_{0}, \Delta\left(y_{n+i-1}, y_{n+j-1}\right) \leq \delta_{0}$, for any $i$, $j \in \mathbb{N}$, we get $\alpha_{n} \leq \alpha\left(\delta_{0}\right)$. Thus, $\delta_{n} \leq \alpha\left(\delta_{0}\right) \delta_{n-1}$, and then $\delta_{n} \leq\left(\alpha\left(\delta_{0}\right)\right)^{n} \delta_{0}$, for all $n \in \mathbb{N}$. which implies that the sequence $\left\{\delta_{n}\right\}$ is convergent to 0 . Furthermore, for all $n, m \in \mathbb{N}$, we have

$\mathscr{D}\left(x_{n}, x_{n+m}\right)=\mathscr{D}\left((S T)^{n} x_{0},(S T)^{n+m} x_{0}\right) \leq \delta_{n}$.
Thus, $\lim _{n, m \rightarrow \infty} \mathscr{D}\left(x_{n}, x_{n+m}\right)=0$, and hence, $\left\{x_{n}\right\}$ is a $\mathscr{D}$-Cauchy sequence. Since $X$ is $\mathscr{D}$-complete, there exists $x^{*} \in X$ such that $\lim _{n \longrightarrow \infty} \mathscr{D}\left(x_{n}, x^{*}\right)=0$.

Step 3. Let $y^{*}=T x^{*}$. Since $\left\{x_{n}\right\}$ is a $G$-increasing sequence and $(X, \mathscr{D}, G)$ is $G$-regular, $x^{*} \in\left[x_{n}\right]_{G}$, for any $n \in \mathbb{N}$. Thus, $x^{*}$ and $S y_{n-1}=x_{n}$ are connected.

In (7), by taking $x=x^{*}$ and $y=y_{n-1}$, we obtain $\Delta\left(T x^{*}, T S y_{n-1}\right) \leq \alpha\left(\mathscr{D}\left(x^{*}, S y_{n-1}\right)\right) \max \left\{\mathscr{D}\left(x^{*}, S y_{n-1}\right)\right.$, $\left.\Delta\left(y_{n-1}, T x^{*}\right), \Delta\left(y_{n-1}, T S y_{n-1}\right)\right\}$. 
As $\lim \sup _{t \rightarrow 0^{+}} \alpha(t)<1$, there exists $k \in[0,1)$ and $N \in \mathbb{N}$ such that for all $n \geq N$, we have $\alpha\left(\mathscr{D}\left(x^{*}, S y_{n-1}\right)\right) \leq k$. Thus,

$$
\Delta\left(y^{*}, y_{n}\right) \leq k \max \left\{\mathscr{D}\left(x^{*}, x_{n}\right), \Delta\left(y_{n-1}, y^{*}\right), \Delta\left(y_{n-1}, y_{n}\right)\right\}
$$

Since $\lim _{n \longrightarrow \infty} \mathscr{D}\left(x_{n}, x^{*}\right)=\lim _{n \longrightarrow \infty} \Delta\left(y_{n-1}, y_{n}\right)=0$, we get $\limsup _{n \rightarrow \infty} \Delta\left(y^{*}, \quad y_{n}\right) \leq k \lim \sup _{n \rightarrow \infty}$ $\Delta\left(y^{*}, y_{n-1}\right)$ and so $\lim \sup _{n \rightarrow \infty} \Delta\left(y^{*}, y_{n}\right)=0$.

As $\quad \lim \sup _{t \rightarrow 0^{+}} \alpha(t)<\inf \{1,(1 / C)\}$, there exists $k^{\prime} \in[0, \inf \{1,(1 / C)\})$ and $N^{\prime} \in \mathbb{N}$ such that for all $n \geq N^{\prime}$, we have $\alpha\left(\Delta\left(y_{n-1}, y^{*}\right)\right) \leq k^{\prime}$. Since $\lim _{n \longrightarrow \infty} \mathscr{D}\left(x_{n}, x^{*}\right)=0$,

$$
\begin{aligned}
\mathscr{D}\left(x^{*}, S y^{*}\right) \leq & C \limsup _{n \longrightarrow \infty} \mathscr{D}\left(x_{n}, S y^{*}\right) \\
= & C \limsup _{n \longrightarrow \infty} \mathscr{D}\left(S y_{n-1}, S T x^{*}\right) \\
\leq & C \limsup _{n \longrightarrow \infty} \alpha\left(\Delta\left(y_{n-1}, y^{*}\right)\right) \max \left\{\mathscr{D}\left(x_{n}, x^{*}\right),\right. \\
& \left.\Delta\left(y_{n-1}, y^{*}\right), \mathscr{D}\left(x^{*}, S y^{*}\right)\right\} \\
\leq & C k^{\prime} \mathscr{D}\left(x^{*}, S y^{*}\right) .
\end{aligned}
$$

We conclude that $\mathscr{D}\left(x^{*}, S y^{*}\right)=0$, since $C k^{\prime}<1$. Then, $x^{*}=S y^{*}$, and so ST $x^{*}=x^{*}$ and $T S y^{*}=y^{*}$.

Since $\left\{x_{n}\right\} \in \mathscr{C}\left(\mathscr{D}, X, x^{*}\right)$ and $\left\{y_{n}\right\} \in \mathscr{C}\left(\mathscr{D}, Y, y^{*}\right)$,

$$
\begin{gathered}
\mathscr{D}\left(x^{*}, x^{*}\right)=0, \\
\Delta\left(y^{*}, y^{*}\right)=0 .
\end{gathered}
$$

Remark 1. In Theorem 2, we can replace the condition $(S T)^{i+1} x_{0} \in\left[(S T)^{i} x_{0}\right]_{G}$ by $(S T)^{i} x_{0} \in\left[(S T)^{i+1} x_{0}\right]_{G}$, for any $i$ in $\mathbb{N}$.

For the uniqueness of the pair $\left(x^{*}, y^{*}\right)$, we establish the following.

Proposition 1. Suppose that there exists another pair $\left(x^{\prime}, y^{\prime}\right)$, satisfying $T x^{\prime}=y^{\prime}$ and $S y^{\prime}=x^{\prime}$.

If $\mathscr{D}\left(x^{\prime}, x^{\prime}\right)<\infty$ and $\Delta\left(y^{\prime}, y^{\prime}\right)<\infty$, then $\mathscr{D}\left(x^{\prime}, x^{\prime}\right)=0$ and $\Delta\left(y^{\prime}, y^{\prime}\right)=0$. Moreover, if $x^{*}$ and $x^{\prime}$ are connected, $\mathscr{D}\left(x^{*}, x^{\prime}\right)<\infty$ and $\Delta\left(y^{*}, y^{\prime}\right)<\infty$ then $\left(x^{\prime}, y^{\prime}\right)=\left(x^{*}, y^{*}\right)$.

Proof. By taking $x=x^{\prime}$ and $y=y^{\prime}$ in (7), we have

$$
\left\{\begin{array}{l}
\mathscr{D}\left(x^{\prime}, x^{\prime}\right)=\mathscr{D}\left(S y^{\prime}, S T x^{\prime}\right) \leq \alpha\left(\Delta\left(y^{\prime}, T x^{\prime}\right)\right) \max \left\{\mathscr{D}\left(x^{\prime}, S y^{\prime}\right), \Delta\left(y^{\prime}, T x^{\prime}\right), \mathscr{D}\left(x^{\prime}, S T x^{\prime}\right)\right\} \\
\Delta\left(y^{\prime}, y^{\prime}\right)=\Delta\left(T x^{\prime}, T S y^{\prime}\right) \leq \alpha\left(\mathscr{D}\left(x^{\prime}, S y^{\prime}\right)\right) \max \left\{\mathscr{D}\left(x^{\prime}, S y^{\prime}\right), \Delta\left(y^{\prime}, T x^{\prime}\right), \Delta\left(y^{\prime}, T S y^{\prime}\right)\right\}
\end{array}\right.
$$

That is,

$$
\left\{\begin{array}{l}
\mathscr{D}\left(x^{\prime}, x^{\prime}\right) \leq \alpha\left(\Delta\left(y^{\prime}, y^{\prime}\right)\right) \Delta\left(y^{\prime}, y^{\prime}\right) \\
\Delta\left(y^{\prime}, y^{\prime}\right) \leq \alpha\left(\mathscr{D}\left(x^{\prime}, x^{\prime}\right)\right) \mathscr{D}\left(x^{\prime}, x^{\prime}\right)
\end{array}\right.
$$

Therefore, we have

$$
\left\{\begin{array}{l}
\left(1-\alpha\left(\Delta\left(y^{\prime}, y^{\prime}\right)\right) \alpha\left(\mathscr{D}\left(x^{\prime}, x^{\prime}\right)\right)\right) \mathscr{D}\left(x^{\prime}, x^{\prime}\right) \leq 0, \\
\left(1-\alpha\left(\Delta\left(y^{\prime}, y^{\prime}\right)\right) \alpha\left(\mathscr{D}\left(x^{\prime}, x^{\prime}\right)\right)\right) \Delta\left(y^{\prime}, y^{\prime}\right) \leq 0
\end{array}\right.
$$

Then, $\mathscr{D}\left(x^{\prime}, x^{\prime}\right)=0$ and $\Delta\left(y^{\prime}, y^{\prime}\right)=0$.

Since $S y^{*}$ and $x^{\prime}$ are connected, by taking $x=x^{\prime}$ and $y=y^{*}$ in (7), we obtain

$$
\left\{\begin{array}{l}
\mathscr{D}\left(S y^{*}, S T x^{\prime}\right) \leq \alpha\left(\Delta\left(y^{*}, T x^{\prime}\right)\right) \max \left\{\mathscr{D}\left(x^{\prime}, S y^{*}\right), \Delta\left(y^{*}, T x^{\prime}\right), \mathscr{D}\left(x^{\prime}, S T x^{\prime}\right)\right\} \\
\Delta\left(T x^{\prime}, T S y^{*}\right) \leq \alpha\left(\mathscr{D}\left(x^{\prime}, S y^{*}\right)\right) \max \left\{\mathscr{D}\left(x^{\prime}, S y^{*}\right), \Delta\left(y^{*}, T x^{\prime}\right), \Delta\left(y^{*}, T S y^{*}\right)\right\} .
\end{array}\right.
$$

That is,

$\left\{\begin{array}{l}\mathscr{D}\left(x^{*}, x^{\prime}\right) \leq \alpha\left(\Delta\left(y^{*}, y^{\prime}\right)\right) \max \left\{\mathscr{D}\left(x^{\prime}, x^{*}\right), \Delta\left(y^{*}, y^{\prime}\right)\right\}, \\ \Delta\left(y^{\prime}, y^{*}\right) \leq \alpha\left(\mathscr{D}\left(x^{\prime}, x^{*}\right)\right) \max \left\{\mathscr{D}\left(x^{\prime}, x^{*}\right), \Delta\left(y^{*}, y^{\prime}\right)\right\} .\end{array}\right.$
Hence,

$$
\left\{\begin{array}{l}
\mathscr{D}\left(x^{*}, x^{\prime}\right) \leq \alpha\left(\Delta\left(y^{*}, y^{\prime}\right)\right) \Delta\left(y^{*}, y^{\prime}\right), \\
\Delta\left(y^{\prime}, y^{*}\right) \leq \alpha\left(\mathscr{D}\left(x^{\prime}, x^{*}\right)\right) \mathscr{D}\left(x^{\prime}, x^{*}\right) .
\end{array}\right.
$$


Therefore, we have $\mathscr{D}\left(x^{\prime}, x^{*}\right)=0$ and $\Delta\left(y^{*}, y^{\prime}\right)=0$, which implies that $\left(x^{\prime}, y^{\prime}\right)=\left(x^{*}, y^{*}\right)$.

Example 1. Let $X=[1,3]$ and $Y=[1,2]$ and two mappings $T: X \longrightarrow Y$ and $S: Y \longrightarrow X$ be defined as follows:

$$
\begin{aligned}
& T x=\frac{x+1}{2}, \quad \text { for any } x \in X, \\
& S y=y+1, \quad \text { for any } y \in Y .
\end{aligned}
$$

Let $\alpha:[0,+\infty) \longrightarrow[0,1)$ such that $\alpha(t)=(2 \sqrt{2} / 3)$. Consider the two mappings $\mathscr{D}: X \times X \longrightarrow[0 ; \infty]$ and $\Delta: Y \times Y \longrightarrow[0 ; \infty]$ defined as follows:

$$
\begin{aligned}
& \mathscr{D}(a, b)=\left|\frac{1}{a}-\frac{1}{b}\right|, \\
& \Delta(c, d)=\frac{1}{\sqrt{2}}\left|\frac{1}{c}-\frac{1}{d}\right| .
\end{aligned}
$$

We can show that $\mathscr{D}$ and $\Delta$ are generalized metrics on $X$ and $Y$, respectively, with constant $C \geq 1$.

Consider the graph $G$ on $X$ such that $V(G)=X$ and

$$
E(G)=\{(x, x): x \in X\} \bigcup\left\{\left(3-\frac{1}{2^{n}}, 3\right): n \in \mathbb{N}\right\} \bigcup\left\{\left(3-\frac{1}{2^{n}}, 3-\frac{1}{2^{n+1}}\right): n \in \mathbb{N}\right\}
$$

We prove that, for any $(x, y) \in X \times Y$, such that $x$ and $S y$ are connected, we have

There are five cases.

Case 1. For $y \in[1,2]$ and $x=S y \in[2,3]$, we have

$$
\begin{aligned}
& \mathscr{D}(S y, S T x)=\left|\frac{1}{x}-\frac{2}{x+3}\right| \leq \frac{2}{3}\left|\frac{1}{x-1}-\frac{2}{x+1}\right| \leq \frac{2 \sqrt{2}}{3} \Delta(y, T x), \\
& \Delta(T x, T S y)=\Delta(T x, T x)=0 .
\end{aligned}
$$

Case 2. For $x=3-\left(1 / 2^{n}\right)$, for any $n \in \mathbb{N}$ and $y=2$, we have

$$
\begin{aligned}
\mathscr{D}(S y, S T x) & =\left|\frac{1}{3-2^{-(n+1)}}-\frac{1}{3}\right| \leq \frac{2 \sqrt{2}}{3}\left|\frac{1}{3-2^{-n}}-\frac{1}{3}\right| \\
& =\frac{2 \sqrt{2}}{3} \mathscr{D}(x, S y), \\
\Delta(T x, T S y) & =\frac{1}{\sqrt{2}}\left|\frac{1}{2-2^{-(n+1)}}-\frac{1}{2}\right| \leq \frac{2 \sqrt{2}}{3}\left|\frac{1}{3-2^{-n}}-\frac{1}{3}\right| \\
& =\frac{2 \sqrt{2}}{3} \mathscr{D}(x, S y) .
\end{aligned}
$$

Case 3. For $x=3$ and $y=2-\left(1 / 2^{n}\right)$, for any $n \in \mathbb{N}$, we have

$$
\begin{aligned}
\Delta(T x, T S y) & =\frac{1}{\sqrt{2}}\left|\frac{1}{2-2^{-(n+1)}}-\frac{1}{2}\right| \leq \frac{2}{3}\left|\frac{1}{2-2^{-n}}-\frac{1}{2}\right| \\
& =\frac{2 \sqrt{2}}{3} \Delta(y, T x) .
\end{aligned}
$$

Case 4. For $x=3-\left(1 / 2^{n}\right)$ and $y=2-\left(1 / 2^{p}\right)$, for any $n, p \in \mathbb{N}$, such that the following can be obtained.

One can see that

$$
\left|\frac{1}{3-\alpha}-\frac{1}{3-\beta}\right| \leq \frac{2}{3}\left|\frac{1}{2-\alpha}-\frac{1}{2-\beta}\right|,
$$

for any $\alpha, \beta \in(0,1]$.

By taking $\alpha=2^{-(n+1)}$ and $\beta=2^{-p}$, we obtain

$$
\left|\frac{1}{3-2^{-(n+1)}}-\frac{1}{3-2^{-p}}\right| \leq \frac{2}{3}\left|\frac{1}{2-2^{-(n+1)}}-\frac{1}{2-2^{-p}}\right| \text {. }
$$

Thus,

$$
\mathscr{D}(S y, S T x) \leq \frac{2 \sqrt{2}}{3} \Delta(y, T x) .
$$

Furthermore, 


$$
\frac{1}{\sqrt{2}}\left|\frac{1}{2-\alpha}-\frac{1}{2-\beta}\right| \leq \frac{2 \sqrt{2}}{3}\left|\frac{1}{3-2 \alpha}-\frac{1}{3-2 \beta}\right|,
$$

for any $\alpha, \beta \in(0,1]$.

By taking $\alpha=2^{-(n+1)}$ and $\beta=2^{-(p+1)}$, we obtain

$$
\frac{1}{\sqrt{2}}\left|\frac{1}{2-2^{-(n+1)}}-\frac{1}{2-2^{-(p+1)}}\right| \leq \frac{2 \sqrt{2}}{3}\left|\frac{1}{3-2^{-n}}-\frac{1}{3-2^{-p}}\right| \text {. }
$$

Thus,

$$
\Delta(T x, T S y) \leq \frac{2 \sqrt{2}}{3} \mathscr{D}(x, S y)
$$

Case 5. For $x=3-\left(1 / 2^{n}\right)$ and $y=2-\left(1 / 2^{p}\right)$, for any $n, p \in \mathbb{N}$, such that $n \geq p+1$. In the same way as Case 4 , we obtain

$$
\begin{aligned}
& \mathscr{D}(S y, S T x) \leq \frac{2 \sqrt{2}}{3} \Delta(y, T x), \\
& \Delta(T x, T S y) \leq \frac{2 \sqrt{2}}{3} \mathscr{D}(x, S y) .
\end{aligned}
$$

Let $x_{0}=2 \in X$, we have $(S T)^{n} x_{0}=3-\left(1 / 2^{n}\right)$, for any $n \in \mathbb{N}$. One can see that

$$
\begin{aligned}
\delta\left(S, T, x_{0}, \mathscr{D}, \Delta\right) & =\sup \left\{\mathscr{D}\left((S T)^{i} x_{0},(S T)^{j} x_{0}\right), \Delta\left(T(S T)^{i} x_{0}, T(S T)^{j} x_{0}\right): i, j \in \mathbb{N}\right\} \\
& =\sup \left\{\mathscr{D}\left(3-\frac{1}{2^{i}}, 3-\frac{1}{2^{j}}\right), \Delta\left(2-\frac{1}{2^{(i+1)}}, 2-\frac{1}{2^{(j+1)}}\right): i, j \in \mathbb{N}\right\} \\
& <\infty
\end{aligned}
$$

and $(S T)^{i+1} x_{0} \in\left[(S T)^{i} x_{0}\right]_{G}$, for all $i \in \mathbb{N}$.

Since $(X, \mathscr{D}, G)$ is $\mathscr{D}$-complete and $G$-regular, then all assumptions of Theorem 2 are satisfied and there exists $\left(x^{*}, y^{*}\right)=(3,2)$ such that $T x^{*}=y^{*}$ and $S y^{*}=x^{*}$.

Remark 2. Theorem 2 extends the main result of Chaira et al. in [3] by considering the graph $G_{\leq}$on $X$ defined by

$$
(x, y) \in E\left(G_{\leq}\right), \quad \text { iff } x \leq y .
$$

The next corollary gives a version of Theorem 2 in standard metric spaces endowed with a digraph.
Corollary 1. Let $(X, d)$ and $(Y, \delta)$ be two metric spaces such that $X$ is endowed with a digraph $G$. Consider a nondecreasing function $\alpha:[0,+\infty) \longrightarrow[0,1)$, such that

$$
\limsup _{t \rightarrow 0^{+}} \alpha(t)<1 \text {. }
$$

Let $T: X \longrightarrow Y$ and $S: Y \longrightarrow X$ be two mappings satisfying the following conditions:

(1) Let

$$
\left\{\begin{array}{l}
d(S y, S T x) \leq \alpha(\delta(y, T x)) \max \{d(x, S y), \delta(y, T x), d(x, S T x)\} \\
\delta(T x, T S y) \leq \alpha(d(x, S y)) \max \{d(x, S y), \delta(y, T x), \delta(y, T S y)\}
\end{array}\right.
$$

for any $x$ in $X$ and $y$ in $Y$, such that $x$ and $S y$ are connected.

(2) $(X, d, G)$ is complete and $G$-regular.

(3) There exists an element $x_{0} \in X$ such that $(S T)^{i+1} x_{0} \in\left[(S T)^{i} x_{0}\right]_{G}$, for all $i \in \mathbb{N}$.

Then, there exists $\left(x^{*}, y^{*}\right) \in X \times Y$, such that $T x^{*}=y^{*}$ and $S y^{*}=x^{*}$; then, ST $x^{*}=x^{*}$ and TS $y^{*}=y^{*}$.

\section{Data Availability}

No data were used to support this study.

\section{Conflicts of Interest}

The authors declare that there are no conflicts of interest regarding the publication of this paper.

\section{References}

[1] S. Banach, "Sur les opérations dans les ensembles abstraits et leur application aux équations intégrales," Fundamenta Mathematicae, vol. 3, no. 1, pp. 133-181, 1922.

[2] B. Fisher, "Fixed point on two metric spaces," Glasnik Matematicki, vol. 16, no. 36, pp. 333-337, 1981. 
[3] K. Chaira, A. Eladraoui, and M. Kabil, "An extension of Fisher fixed point theorem in partially ordered generalized metric spaces," Malaya Journal of Matematik, vol. 5, no. 4, pp. 647652, 2017.

[4] S. Czerwik, "Contraction mappings in b-metric spaces," Acta Mathematica et Informatica Universitatis Ostraviensis, vol. 1, no. 1, pp. 5-11, 1993.

[5] P. Hitzler and A. K. Seda, "Dislocated topologies," Journal of Electrical Engineering, vol. 51, no. 12, pp. 3-7, 2000.

[6] E. Karapinar and P. Salimi, "Dislocated metric space to metric spaces with some fixed point theorems," Fixed Point Theory and Applications, vol. 2013, no. 222, pp. 1-19, 2013.

[7] M. Jleli and B. Samet, "A generalized metric space and related fixed point theorems," Fixed Point Theory and Applications, vol. 2015, no. 61, pp. 1-14, 2015.

[8] N. Hussain, Z. D. Mitrović, and S. Radenović, "A common fixed point theorem of Fisher in b-metric spaces," Revista de la Real Academia de Ciencias Exactas, Físicas y Naturales. Serie A. Matemáticas, vol. 113, no. 2, pp. 949-956, 2019.

[9] V. Parvaneh, N. Hussain, and M. A. Kutbi, "Some fixed point results in extended parametric $b$-metric spaces with application to integral equations," Journal of Mathematical Analysis, vol. 10, no. 5, pp. 14-33, 2019.

[10] T. Chaiporn and A. Khemphet, "Coincidence point theorems for $(\alpha, \beta, \gamma)$-contraction mappings in generalized metric spaces," International Journal of Mathematics and Mathematical Sciences, vol. 2018, Article ID 4053478, 7 pages, 2018.

[11] E. Karapinar, B. Samet, and D. Zhang, "Meir-Keeler type contractions on JS-metric spaces and related fixed point theorems," Journal of Fixed Point Theory and Applications, vol. 20, no. 2, pp. 60-78, 2018.

[12] I. Altun and B. Samet, "Pseudo Picard operators on generalized metric spaces," Applicable Analysis and Discrete Mathematics, vol. 12, no. 2, pp. 389-400, 2018.

[13] T. Senapati, K. D. Lakshmi, and D. D. Diana, "Extensions of Ćirić and Wardowski type fixed point theorems in D-generalized metric spaces," Fixed Point Theory and Applications, vol. 2016, no. 33, pp. 1-14, 2016.

[14] J. Jachymski, "The contraction principle for mappings on a metric space with a graph," Proceedings of the American Mathematical Society, vol. 136, no. 4, pp. 1359-1373, 2007.

[15] B. Samet and M. Turinici, "Fixed point theorems on a metric space endowed with an arbitrary binary relation and applications," Communications in Mathematical Analysis, vol. 13, no. 2, pp. 82-97, 2012.

[16] K. Chaira, M. Kabil, and A. Kamouss, "Common fixed points in generalized metric spaces with a graph," Journal of Mathematics, vol. 2019, Article ID 8956083, 4 pages, 2019.

[17] K. Chaira, A. Eladraoui, M. Kabil, and A. Kamouss, "Kannan fixed point theorem on generalized metric space with a graph," Applied Mathematical Sciences, vol. 13, no. 6, pp. 263-274, 2019.

[18] R. Espinola and W. A. Kirk, "Fixed point theorems in R-trees with applications to graph theory," Topology and Its Applications, vol. 153, no. 7, pp. 1046-1055, 2006.

[19] J. A. Bondy and U. S. R. Murty, "Graph theory," in Graduate Texts in Mathematics, Vol. 244, Springer, New York, USA, 2008.

[20] W. D. Wallis, A Beginner's Guide to Graph Theory, Springer Science and Business Media, Berlin, Germany, 2010. 